Vol. $8 \mid 20$ | International Journal of

№2 21 Medical and Surgical Sciences

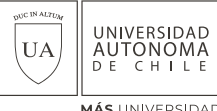

Article

FENOTIPOS GLÍNICOS DE LA ENFERMEDAD

PULMONAR OBSTRUGTIVA GRÓNIGA EN LOS

PACIENTES ATENDIDOS EN EL HOSPITAL

Neumológico de LA Habana

\title{
Clinical phenotypes of chronic obstructive pulmonary disease in patients treated at the Hospital Neumológico de La Habana
}

\author{
ILIS Herrera-Monterrey (D) \\ Hospital Clínico Quirúrgico Provincial Abel Santamaría Cuadrado. Pinar del Río, Cuba.
}

DONEL GONZÁLEZ-DÍAz (1)

Hospital Clínico Quirúrgico Hermanos Ameijeiras. La Habana, Cuba.

Otilia María Terry-Valles (i)

Hospital Neumológico de Referencia Nacional Benéfico Jurídico. La Habana, Cuba.

DrIALIS DÍAZ-GARRIDo (1)

Hospital Clínico Quirúrgico Hermanos Ameijeiras. La Habana, Cuba.

Sergio Fernández-García (i)

Hospital Clínico Quirúrgico Hermanos Ameijeiras. La Habana, Cuba.

Autor para la correspondencia: Dr. Donel González

Hospital Clínico Quirúrgico Hermanos Ameijeiras. La Habana, Cuba.

Mail:dmachual@gmail.com

Recibido: $21 / 12 / 2020$

Aceptado: 17/02/2021

\section{RESUMEN}

Introducción: La identificación de los fenotipos clínicos son claves en la modulación de la expresión clínica, para un tratamiento integrado de la EPOC. Objetivos: Caracterizar los fenotipos clínicos de la EPOC en los pacientes atendidos en el Hospital Neumológico Benéfico Jurídico. Métodos: Se realizó un estudio observacional descriptivo retrospectivo, en 172 pacientes con diagnóstico de EPOC, en el Hospital Neumológico Benéfico Jurídico durante el año 2017.Resultados: El $38,4 \%$ de los pacientes tenían edad entre 70-79 años. Del total de pacientes, el 54,6 \% eran del sexo masculino. El 52,9 \% eran fumadores activos y el 41,3\% exfumadores. Aunque las 
diferencias no fueron significativas, la edad avanzada y el sexo masculino fueron más frecuentes en el fenotipo enfisematoso agudizador y agudizador bronquítico crónico. El tabaquismo activo fue más frecuente en el fenotipo enfisematoso agudizador. Todos los pacientes con el fenotipo agudizador bronquítico crónico tuvieron dos o más exacerbaciones, mientras que el enfisematoso agudizador se relacionó con una severidad grave de la EPOG (46,7\%). Conclusiones: El sexo masculino y la edad avanzada muestran una tendencia a relacionarse con el fenotipo enfisematoso agudizador y agudizador bronquítico crónico, mientras que el tabaquismo activo es más frecuente en el fenotipo enfisematoso agudizador. El fenotipo agudizador bronquítico crónico se relaciona con mayores exacerbaciones y el enfisematoso agudizador con una mayor severidad de la EPOG.

Palabras clave: EPOG, fenotipos clínicos de la enfermedad pulmonar obstructiva crónica; epidemiología clínica.

\section{Introducción}

La enfermedad pulmonar obstructiva crónica (EPOC) se define desde el punto de vista funcional como la presencia de una alteración obstructiva de la ventilación, permanente o parcialmente reversible, dado por disminución del volumen espiratorio forzado en el primer segundo $\left(\mathrm{FEV}_{1}\right)$ y disminución del cociente $\mathrm{FEV}_{1}$ /capacidad vital forzada (FVC). Existe una gran variedad de factores de riesgo o predisponentes para la EPOC, entre los cuales se encuentra la exposición al humo del tabaco, así como la exposición laboral o profesional a humos y a polvos. También se incluye las infecciones respiratorias graves a temprana edad, así como el tabaquismo pasivo, la exposición al humo de leña y la contaminación ambiental. (Casas et al., 2017).

Debido a la tendencia a personalizar lo más posible el tratamiento del paciente de acuerdo con la característica de la enfermedad, se ha creado una clasificación fenotípica de la EPOC. El fenotipo puede ser un atributo simple o una combinación de atributos de la enfermedad, que describa una diferencia entre individuos con EPOC. Pueden relacionarse con características clínicas significativas, como los síntomas, exacerbaciones, respuesta a la terapia, velocidad de la progresión, tendencia a la exacerbación o ser cuadros mixtos de asma y EPOC. (Miravitlles et al., 2012)

La Sociedad Española de Patología Respiratoria (SEPAR) ha aplicado las características fenotípicas más importantes, estableciendo una nueva clasificación y orientación terapéutica. La clasificación establece el término agudizador en vez de exacerbación y propone 5 fenotipos: el no agudizador (A), el agudizador enfisematoso $(\mathrm{C})$, el agudizador bronquítico crónico (D) y el mixto asma- EPOC (B), que se cataloga así por su alta reversibilidad funcional con el broncodilatador y eosinofilia en el esputo. (Miravitlles et al., 2014)

En la Unión Europea, se estima que los costos directos totales de las enfermedades respiratorias representan aproximadamente el $6 \%$ del total de la factura sanitaria, correspondiendo a la EPOC más de la mitad ( $56 \%$ ) de este gasto, que equivale a 38600 millones de euros. Representa un elevado coste sanitario y constituye la cuarta causa de muerte en los países de este entorno y, según estimaciones de la Organización Mundial de la Salud, será la tercera en el año 2030. (De Abajo et al., 2017; Morales et al., 2015)

En América Latina, se estima que en Colombia el estudio PREPOCOL determinó la prevalencia del EPOC del 8,9 \% en mayores de 40 años siendo la séptima causa de muerte en el país. En Brasil la EPOC tuvo una prevalencia de 19,7\% . (Vinaccia et al., 2017) 
En Cuba, se desconoce la situación real, en algunas investigaciones locales se ha llegado a estimar alrededor del 13 al $18 \%$. Al cierre del 2019 sucedieron 4079 muertes por enfermedades crónicas de las vías respiratorias inferiores, constituyendo la sexta causa de muerte para todas las edades con una tasa ajustada anual de mortalidad de 38,7 por cada 100 mil habitantes entre los varones y 33,8 por cada 100 mil habitantes entre las mujeres. (Robainas et al., 2020)

En el Hospital Neumológico Benéfico Jurídico, en la provincia La Habana, Cuba, la morbilidad por EPOC en consulta externa fue de 4521 en los años comprendidos entre 2015-2017, lo que representa el 15,2\%. Fueron ingresados 403 pacientes por esta causa $(9,8 \%)$.

Se trata de una enfermedad infradiagnosticada, infratratada y con una elevada morbimortalidad, y supone un problema de salud pública de gran magnitud. La aparición de esta enfermedad avanza con la edad entre la población adulta. A medida que la población vaya envejeciendo, la prevalencia de la EPOC se incrementará, y como resultado cada vez más pacientes necesitarán atención sanitaria. Si se tiene en cuenta que en Cuba casi un 20 \% de la población está compuesta por individuo de 60 años y más, además en los años venideros se incrementará el envejecimiento de la población, puede tenerse idea de la magnitud del problema por lo comentado anteriormente.

La investigación tiene el propósito de contribuir con la identificación de los fenotipos de la EPOC, que van a ser claves en la modulación de la expresión clínica, para un tratamiento integrado, el objetivo fundamental que es tratar al paciente y no solo una enfermedad, que permitirá la utilización de medidas tempranas y durables, en el restablecimiento en función de evitar el deterioro de la calidad de vida, prolongar la esperanza de vida en estos pacientes en beneficio de los pacientes con EPOC que acuden a consultas, especialmente el más grave que requiere una mayor atención especializada, resulta evidente que en el futuro va a suponer un reto para el neumólogo.

\section{Material y métodos}

Se realizó un estudio observacional descriptivo retrospectivo, en pacientes con diagnóstico de EPOC, en el Hospital Neumológico Benéfico Jurídico durante el año 2017.

El universo de estudio fue la totalidad de pacientes con diagnóstico de EPOC (n=326). La muestra quedó constituida por $(\mathrm{n}=172)$ pacientes. Se tuvo en cuenta para incluir en el estudio aquellos pacientes con diagnóstico de EPOC, confirmados por espirometría forzada y de ambos sexos, mayores de 18 años que fueron diagnosticados e ingresados en el Hospital Benéfico Jurídico durante el año 2017. Se excluyeron pacientes que no presentaron una historia clínica completa, que comprometió el desarrollo de la investigación.

La clasificación de los fenotipos clínicos de la EPOC se basó según la establecida por la Guía Española (GesEPOC) en: Fenotipo no agudizador cuando presenta menos de 2 agudizaciones/año con enfisema o bronquitis crónica, fenotipo agudizador todo paciente con EPOC que presente 2 o más agudizaciones moderadas o graves al año, se considera que 4 semanas al término del tratamiento de la agudización o 6 semanas desde el inicio en casos sin tratamiento. Se definió fenotipo enfisema agudizador cuando incluye las condiciones de agudización y además presenta disnea e intolerancia al ejercicio como síntomas predominantes, el fenotipo bronquítico agudizador cuando incluye las condiciones de agudización y presenta tos crónica con expectoración como síntomas predominantes, el fenotipo mixto asma-EPOC se definió que debe cumplir al menos 2 criterios mayores o 1 mayor y 2 menores teniendo en cuenta los criterios establecidos, los mayores son: prueba broncodilatadora 
muy positiva (incremento del $\mathrm{FEV}_{1}$ mayor $15 \%$ y mayor $400 \mathrm{ml}$ ), eosinofilia en esputo y antecedentes personales de asma, y los menores son: antecedentes personales de atopia, prueba broncodilatadora positiva en al menos 2 ocasiones (incremento del $\mathrm{FEV}_{1}$ mayor $12 \%$ y mayor $200 \mathrm{ml}$ ) y cifras elevadas de Inmunoglobulina $\mathrm{E}(\mathrm{IgE})$.

Se definió el número de exacerbaciones al aumento transitorio de la gravedad de los síntomas durante un período de tiempo equivalente a un año.

La severidad de la EPOG se definió según el grado de obstrucción, dado por el $\mathrm{FEV}_{1}$, obtenido en la espirometría forzada, categorizando la gravedad de la limitación del flujo aéreo de la EPOC, establecida en la Global Initiative for Chronic Obstructive Lung Disease (GOLD) como: leve $\left(\mathrm{FEV}_{1}>70 \%\right.$ y $<80 \%)$, moderada $\left(\mathrm{FEV}_{1}>50 \% \mathrm{y}<70 \%\right)$, severa $\left(\mathrm{FEV}_{1}>30 \% \mathrm{y}<50 \%\right)$ y muy severa $\left(\mathrm{FEV}_{1}<\right.$ $30 \%$ ).

La información obtenida fue depositada en una base de datos, a través de la hoja de cálculo Excel Microsoft Office de Windows 10, además fue procesada para los cálculos y análisis posteriores mediante el sistema SPSS versión 23.

Para el análisis estadístico las variables cualitativas se agruparon en números absolutos y por cientos, las cuantitativas en media y desviación estándar. Se utilizaron los siguientes estadígrafos: el Chi cuadrado de Pearson para determinar la posible asociación entre variables cualitativas y la $T$ Student para variables cuantitativas. Se definió la presencia de asociación significativa para valores de $\mathrm{p}<0,05$. Se diseñaron tablas para el mejor análisis e interpretación de los resultados obtenidos.

La investigación respetó los postulados de la ética que aparecen en la Declaración de Helsinki. La información se mantuvo con la más absoluta fidelidad tratada de forma confidencial y con fines puramente científicos. Se solicitó la autorización de la Dirección del Hospital, a través del Consejo Científico y la Comisión de Ética Médica.

Tras la identificación del paciente en fenotipo Mixto (ASMA-EPOG) según criterios propuestos por la GesEPOC se cuenta con criterios mayores y menores como se expone anteriormente. Por no disponibilidad de los recursos para realizar el conteo de eosinófilos en esputo y la dosificación de la IgE son sesgos a la investigación. Pero para el diagnóstico del fenotipo Mixto (Asma-EPOC) solo bastan dos criterios mayores o un criterio mayor y dos menores, por lo tanto, no interfieren en la realización del estudio.

\section{Resultados}

En la Tabla I se observa un predominio de los pacientes con edad entre 50-59 años en el grupo de no agudizadores $(27,5 \%$ ), mientras que en el grupo mixto y enfisematoso agudizador predominaron los grupos de 60-69 años (43,2 \% y 46,7 \% respectivamente) y en el no agudizador el grupo de 70-79 años $(42,5 \%)$. Estas diferencias fueron significativas desde el punto de vista cualitativo. No obstante, al analizar la media y desviación estándar de cada uno de los grupos de comparación de acuerdo con el fenotipo clínico las diferencias no fueron significativas respecto a la edad. 
Tabla I.

Distribución de pacientes según fenotipos clínicos y grupos de edad.

\begin{tabular}{ccccc}
\hline \multirow{2}{*}{ Grupos de edad } & \multicolumn{4}{c}{ Fenotipos } \\
\cline { 2 - 5 } & $\begin{array}{c}\text { No agudizador } \\
\mathbf{n = 4 0}\end{array}$ & $\begin{array}{c}\text { Mixto } \\
\mathbf{n = 3 7}\end{array}$ & $\begin{array}{c}\text { EA } \\
\mathbf{n = 6 0}\end{array}$ & $\begin{array}{c}\text { ABC } \\
\mathbf{n = 3 5}\end{array}$ \\
\hline $40-49$ años & $1(2,5 \%)$ & $2(5,4 \%)$ & $1(1,7 \%)$ & $0(0,0 \%)$ \\
$50-59$ años & $11(27,5 \%)$ & $6(16,2 \%)$ & $3(5,0 \%)$ & $5(14,3 \%)$ \\
$60-69$ años & $6(15,0 \%)$ & $16(43,2 \%)$ & $28(46,7 \%)$ & $11(31,4 \%)$ \\
$70-79$ años & $17(42,5 \%)$ & $11(29,7 \%)$ & $22(36,7 \%)$ & $16(45,7 \%)$ \\
80 años y más & $5(12,5 \%)$ & $2(5,4 \%)$ & $6(10,0 \%)$ & $3(8,6 \%)$ \\
Edad D.E ${ }^{\text {a }}$ & $68,5 \pm 10,5$ & $66,1 \pm 9,8$ & $69,1 \pm 8,5$ & $69,3 \pm 8,6$ \\
\hline
\end{tabular}

$\mathrm{X}^{2}=21,061 ; \mathrm{p}=0,049 ;{ }^{\text {a }}$ ANOVA $\mathrm{p}=0,396$; EA: Enfisema agudizador; ABC: Agudizador bronquítico crónico;

D.E: Desviación estándar.

De acuerdo con la distribución de género, en la Tabla II se observa que el porcentaje de mujeres fue más frecuente en el fenotipo Mixto (56,8 \%) mientras que el sexo masculino fue más frecuente en el fenotipo agudizador bronquítico crónico (62,9 \%). Para el no agudizador y enfisematoso agudizador también se encontró un ligero predominio del sexo masculino, aunque las diferencias no fueron significativas $(p=0,393)$.

Tabla II.

Distribución de pacientes según fenotipos clínicos y sexo.

\begin{tabular}{cccccc}
\hline \multirow{2}{*}{ Sexo } & \multicolumn{5}{c}{ Fenotipos } \\
\cline { 2 - 5 } & $\begin{array}{c}\text { No agudizador } \\
\mathbf{n = 4 0}\end{array}$ & $\begin{array}{c}\text { Mixto } \\
\mathbf{n = 3 7}\end{array}$ & $\begin{array}{c}\text { EA } \\
\mathbf{n = 6 0}\end{array}$ & $\begin{array}{c}\text { ABC } \\
\mathbf{n = 3 5}\end{array}$ & $\begin{array}{c}\text { Total } \\
\mathbf{n = 1 7 2}\end{array}$ \\
\hline Femenino & $18(45,0 \%)$ & $21(56,8 \%)$ & $26(43,3 \%)$ & $13(38,1 \%)$ & $78(45,3 \%)$ \\
Masculino & $22(55,0 \%)$ & $16(43,2 \%)$ & $34(56,7 \%)$ & $22(62,9 \%)$ & $94(54,6 \%)$ \\
\hline
\end{tabular}

$\mathrm{X}^{2}=2,993 ; \mathrm{p}=0,393$; EA: Enfisema agudizador; ABC: Agudizador bronquítico crónico.

Respecto al tabaquismo, la Tabla III muestra un mayor porcentaje de fumadores activos en el fenotipo enfisematoso agudizador (65,0 \%), mientras que el mayor por ciento de exfumadores se encontró en el fenotipo agudizador bronquítico crónico $(57,1 \%$ ). Los no fumadores fueron más frecuentes con el fenotipo no agudizador. No obstante, las diferencias no llegaron a ser estadísticamente significativas $(\mathrm{p}=0,079)$.

Tabla III.

Distribución de pacientes según fenotipos clínicos y tabaquismo.

\begin{tabular}{ccccc}
\hline \multirow{2}{*}{ Tabaquismo } & \multicolumn{4}{c}{ Fenotipos } \\
\cline { 2 - 5 } & $\begin{array}{c}\text { No agudizador } \\
\mathbf{n = 4 0}\end{array}$ & $\begin{array}{c}\text { Mixto } \\
\mathbf{n = 3 7}\end{array}$ & $\begin{array}{c}\text { EA } \\
\mathbf{n = 6 0}\end{array}$ & $\begin{array}{c}\text { ABC } \\
\mathbf{n}=\mathbf{3 5}\end{array}$ \\
\hline Fumador & $19(47,5 \%)$ & $20(54,1 \%)$ & $39(65,0 \%)$ & $14(40,0 \%)$ \\
Exfumador & $16(40,0 \%)$ & $16(43,2 \%)$ & $18(30,0 \%)$ & $20(57,1 \%)$ \\
No fuma & $5(12,5 \%)$ & $1(2,7 \%)$ & $3(5,0 \%)$ & $1(2,9 \%)$ \\
\hline
\end{tabular}

$\mathrm{X}^{2}=1$ 1,310; p=0,079; EA: Enfisema agudizador; ABC: Agudizador bronquítico crónico 
De acuerdo con el número de exacerbaciones, la Tabla IV muestra que la totalidad de los casos con fenotipo agudizador bronquítico crónico tuvieron dos o más exacerbaciones. Este porcentaje también fue significativamente mayor en el fenotipo enfisematoso agudizador respecto a aquellos con fenotipo mixto y de estos respecto a los no agudizadores (88,3\% frente a $62,2 \%$ frente a $2,5 \%$ respectivamente). Por el contrario, el 97,5\% de los no agudizadores tuvieron menos de dos exacerbaciones durante el año evaluado. Estas diferencias fueron muy significativas.

\section{Tabla IV.}

Distribución de pacientes según fenotipos clínicos y número de exacerbaciones.

\begin{tabular}{ccccc}
\hline \multirow{2}{*}{ Exacerbaciones } & \multicolumn{4}{c}{ Fenotipos } \\
\cline { 2 - 5 } & No agudizador $\mathrm{n}=40$ & Mixto $\mathrm{n}=37$ & EA n=60 & ABC n=35 \\
\hline Menos de dos & $40(100,0 \%)$ & $14(37,8 \%)$ & $7(11,7 \%)$ & $0(0,0 \%)$ \\
Dos o más & $0(0,0 \%)$ & $23(62,2 \%)$ & $53(88,3 \%)$ & $35(100,0 \%)$ \\
\hline
\end{tabular}

$\mathrm{X}^{2}=102,174 ; \mathrm{p}<0,0001$

Como se observa en la Tabla V, el porciento de pacientes con EPOG grave $(46,7 \%$ frente a 35,1 $\%$ frente a $28,6 \%$ frente a $10,0 \%$ por ese orden) y muy grave $(15,0 \%$ frente a $8,1 \%$ frente a $5,7 \%$ frente a $0,0 \%$ respectivamente) fue significativamente mayor en el grupo enfisematoso agudizador respecto al mixto, el agudizador bronquítico crónico y, por último, el no agudizador.

Tabla V.

Distribución de pacientes según severidad de la EPOG y fenotipos encontrados.

\begin{tabular}{ccccc}
\hline \multirow{2}{*}{ Severidad } & \multicolumn{4}{c}{ Fenotipos } \\
\cline { 2 - 5 } & No agudizador $\mathrm{n}=40$ & Mixto $\mathrm{n}=37$ & EA n=60 & ABC n=35 \\
\hline Leve & $27(67,5 \%)$ & $8(21,6 \%)$ & $2(3,3 \%)$ & $7(20,0 \%)$ \\
Moderada & $9(22,5 \%)$ & $13(35,1 \%)$ & $21(35,0 \%)$ & $16(45,7 \%)$ \\
Grave & $4(10,0 \%)$ & $13(35,1 \%)$ & $28(46,7 \%)$ & $10(28,6 \%)$ \\
Muy Grave & $0(0,0 \%)$ & $3(8,1 \%)$ & $9(15,0 \%)$ & $2(5,7 \%)$ \\
\hline
\end{tabular}

$\mathrm{X}^{2}=60,019 ; \mathrm{p}<0,0001$

\section{Discusión}

Respecto a la edad, en un estudio realizado en Santiago de Cuba, se reportó un predominio de los grupos de edad entre 50-59 (45,8 \%) y 60-69 años (35,4\%) respectivamente, similar a los resultados de esta investigación. (Legrá et al., 2014).

En una investigación realizada en el mismo Hospital Neumológico de La Habana, que si bien se utilizó un enfoque clínico-epidemiológico diferente al que se expone en este estudio, expusieron que la mayor incidencia estuvo en los pacientes mayores 60 años para ambos sexos. (Robainas del Pino et al., 2020) y en el presente estudio, los pacientes se caracterizaron por ser del sexo femenino, con una edad mayor de 60 años. Si bien la EPOC es una enfermedad con elevada prevalencia, morbilidad y mortalidad tradicionalmente asociada al sexo masculino, en los últimos estudios epidemiológicos se refleja un aumento significativo de la prevalencia de la EPOC en mujeres. (Ntritsos et al., 2018) 
Investigaciones internacionales y foráneas difieren en cuanto al sexo. En varios estudios coinciden en una distribución por sexo, con predominio de hombres, con el promedio de edad de 66,5 \pm 14 años, además, reflejaron que la prevalencia total de la EPOC fue de 15,8 \% (IC $95 \%$ : 13,2-18,4), con una prevalencia en hombres del 18,9\% (IC 95 \%: 15,4-22,4). (De Abajo et al., 2017; Martínez et al., 2016; Legrá et al., 2014 ). Por lo contrario, en un estudio retrospectivo en Perú, realizado en 140 pacientes con EPOC, la diferencia entre hombres y mujeres fue menor $(52,9 \%$ frente a 47,1 \%). (Vidalón-Soldevilla et al., 2011)

Sin lugar a duda, el hábito de fumar constituye el factor de riesgo más importante para el desarrollo de la EPOC. Respecto al tabaquismo, en el presente estudio la totalidad de los pacientes estaban relacionados con este factor de riesgo, de los cuales la mayoría eran fumadores activos, aunque con menos de 10 cigarrillos diarios. En estudios de cohortes prospectivos se estima que el riesgo absoluto de desarrollar EPOC entre fumadores está entre el 25 y el $30 \%$. Además, se ha demostrado que el riesgo es proporcional al consumo acumulado de tabaco, de tal forma que el riesgo pasa del $26 \%$ en los fumadores de 15-30 paquetes al año, al $51 \%$ en los fumadores de más de 30 paquetes al año. (Sáenz., 2017; Ruiz et al., 2017; Agustí et al 2018)

En el presente estudio, el fenotipo clínico más frecuente fue el enfisematoso agudizador. Difiere a estos resultados, una investigación española donde el fenotipo más frecuente fue el no agudizador (62\%), seguido por el agudizador bronquítico crónico (22,0\%), el enfisematoso agudizador $(10,0 \%$ y en solo un 6,0\% el Mixto. (Izquierdo et al., 2017)

En el presente trabajo, el fenotipo no agudizador fue el segundo en frecuencia $(23,3 \%)$. En un estudio prospectivo observacional sobre 2138 pacientes con EPOC moderada-grave seguidos durante tres años, el $23 \%$ de los pacientes no sufrieron ninguna agudización, porciento muy similar al encontrado en este trabajo. (Agusti et al., 2014)

La prevalencia del fenotipo mixto es desconocida, pero existen diferentes estimaciones de su importancia en el contexto de la EPOC. Hay estudios que han cifrado la prevalencia del fenotipo mixto (identificado por eosinofilia en el esputo) en pacientes con EPOG en el $38 \%$ de los casos, asociado de forma directa con la respuesta terapéutica a los corticoides inhalados. Si se emplea como referencia la prueba broncodilatadora, el 31,5\% de los pacientes identificados con EPOC en el estudio epidemiológico EPI-SCAN tenían una prueba positiva. Basados en esos resultados se ha concluido que, en conjunto, entre el 20 y el $40 \%$ de pacientes con EPOC pueden ser portadores de un fenotipo mixto. (García et al., 2017; Casas et al., 2017)

El fenotipo mixto es tema de discusión actual, por parte del gremio médico ya que, tanto la EPOC como el asma bronquial, son enfermedades inflamatorias crónicas que afectan a la vía aérea y se caracterizan esencialmente por la presencia de obstrucción bronquial, que en ocasiones el diagnóstico diferencial se hace difícil por la similitud de los síntomas respiratorios. Existen criterios como se mencionan anteriormente, para poder llegar a un diagnóstico certero. La escases de estudios internacionales, y las críticas de los pocos que existen, han aportado un consenso paa establecer las características clínicas del fenotipo con un comportamiento diferencial. Por tanto la no presencia de todos los criterios, no excluyen que el enfermo padezca este fenotipo clínico. ((Miravitlles et al., 2012).

La prevalencia de los fenotipos de la EPOC varía de un servicio u hospital, en los servicios de Medicina Interna en 8 hospitales de la provincia de Alicante se obtuvo una prevalencia de 44 $\%, 36,4 \%, 15,2 \%$ y $4,0 \%$ de los fenotipos no reagudizador, fenotipo agudizador con bronquitis crónica, fenotipo agudizador con enfisema y mixto, respectivamente. Sin embargo, en los servicios de 
neumología el 43,2 \% presentó fenotipo enfisematoso, el 44,7 \% bronquitis crónica y solo el 12,1% fenotipo mixto. (Rubio et al., 2017)

En la edad media fue significativamente mayor en el grupo con bronquitis crónica respecto al enfisematoso agudizador y de estos respecto al fenotipo mixto (74,6 frente a 70,1 frente a 69,1 años, respectivamente), lo que de cierta forma coincide con los resultados de este trabajo, ya que si bien no se encontraron diferencias significativas, el fenotipo mixto mostró la menor edad media y los fenotipos enfisematoso agudizador y agudizador bronquítico crónico fueron los de mayor edad. (Golpe et al., 2014)

Otro hallazgo reportado fue que el fenotipo agudizador, ya fuese bronquítico o enfisematoso, fue significativamente mayor a medida que avanzaron los grupos de edad, sobre todo para las mujeres. (Cosio et al., 2016)

Por el contrario, en otros estudios, ni la edad media al momento del diagnóstico ni al momento de inclusión en el estudio, fueron significativamente diferentes en los grupos de fenotipos clínicos comparados. (Reiger et al., 2018; Kania et al.,2018)

De acuerdo con la distribución por sexo, el porciento de hombres fue significativamente mayor en el grupo con bronquitis crónica respecto al enfisematoso agudizador y mucho mayor de estos respecto al fenotipo mixto (85,6 \% frente a 80,2 \% frente a 37,7 \%, respectivamente). (Golpe et al., 2014). Estos resultados coinciden con el presente trabajo, en el que el sexo masculino mostró una tendencia a relacionarse con el fenotipo enfisematoso agudizador y agudizador bronquítico crónico.

De manera similar, en un estudio realizado en Austria, el sexo masculino fue mucho más frecuente en el fenotipo enfisematoso agudizador $(71,7 \%$ ) y agudizador bronquítico crónico $(72,1 \%)$, respecto al no agudizador $(64,3 \%$ ) y al mixto (45,5\%), respectivamente, aunque las diferencias no llegaron a ser estadísticamente significativas, quizás porque el tamaño de la muestra no fue muy grande. (Reiger et al., 2018)

De acuerdo con el tabaquismo, se detectó que el fumador activo era más frecuente en el fenotipo enfisematoso agudizador, aunque la asociación no llegó a ser significativa. Contrario a estos resultados encontraron un mayor por ciento de pacientes fumadores activos en el grupo mixto y de exfumadores en las dos variantes de agudizadores. (Kania et al.,2018)

En el estudio ADEPOCLE las reagudizaciones variaron en función del fenotipo, siendo más frecuentes en el fenotipo agudizador con bronquitis crónica en el $40 \%$, el fenotipo agudizador con enfisema $(27 \%$ ) y el fenotipo mixto (23\%), por ese orden de frecuencia. (De Abajo et al., 2017). En otra investigación relacionando los volúmenes pulmonares tanto el $\mathrm{FEV}_{1}$ como la FVC fueron significativamente menores en los agudizadores bronquíticos y con enfisema, mientras que el número de exacerbaciones fue mayor en el bronquítico crónico. (Kania et al.,2018) Estos resultados coinciden con los encontrados en el presente trabajo, en el que el fenotipo agudizador bronquítico crónico se relacionó con mayor número de exacerbaciones y el enfisematoso agudizador con una mayor severidad de la EPOC.

En el estudio PLATINO se señaló que el fenotipo bronquítico crónico tiene una peor función pulmonar, peor estado de salud general, así como mayor número de síntomas respiratorios, limitación a la actividad física y mayor número de exacerbaciones respecto a los otros fenotipos. (de Oca et al., 2012) 
De igual manera, en otro reporte de series, se encontró un porciento significativamente mayor de pacientes con dos o más agudizaciones al año en el grupo bronquítico crónico (49,1\%). No obstante, difiere en que el fenotipo Mixto le siguió en orden de frecuencia $(37,7 \%)$ y por último el enfisematoso agudizador (25,0\%). (Golpe et al., 2014)

Otro estudio reciente ha informado de que el fenotipo agudizador con enfisema grave, hiperinsuflación pulmonar severa y niveles disminuidos de proteína en suero es un predictor independiente de las exacerbaciones frecuentes de la EPOG porque se encontró una media de exacerbaciones y hospitalizaciones en el año previo, significativamente mayor en el grupo de agudizadores (sobre todo los enfisematosos) respecto al mixto y, por supuesto, los no agudizadores. (Rubio et al., 2017)

El reconocimiento de un fenotipo predominante puede ayudar a guiar la mejor opción terapéutica para un solo paciente, y este es el fundamento del nuevo enfoque de las guías orientadas al fenotipo. Sin embargo, las propuestas se basan en el consenso de expertos basado en la revisión de la literatura y la experiencia clínica, ya que no existe evidencia que demuestre que un enfoque de fenotipo sea mejor que el enfoque actualmente recomendado basado en la función pulmonar, los síntomas y las exacerbaciones.

El presente trabajo demuestra que una clasificación basada en el fenotipo es fácil de aplicar en la mayoría de los pacientes con EPOC atendidos en los centros hospitalarios, muestra la distribución y algunas características demográficas y clínicas de los pacientes clasificados por este algoritmo clínico.

\section{Conclusión}

El sexo masculino y la edad avanzada muestran una tendencia a relacionarse con el fenotipo enfisematoso agudizador y agudizador bronquítico crónico, mientras que el tabaquismo activo es más frecuente en el fenotipo enfisematoso agudizador. El fenotipo agudizador bronquítico crónico se relaciona con mayores exacerbaciones y el enfisematoso agudizador con una mayor severidad de la EPOC.

\section{Aspectos éticos}

La investigación respetó los postulados de la ética que aparecen en la Declaración de Helsinki. La información se mantuvo con la más absoluta fidelidad tratada de forma confidencial y con fines puramente científicos. Se solicitó la autorización de la Dirección del Hospital, a través del Consejo Científico y la Comisión de Ética Médica

\section{Conflictos de interés}

Los autores declaran no tener conflictos de intereses

\section{Financiamiento}

No. 


\section{Referencias}

Agusti A, Calverley PMA, Celli B, Coxson HO, Edwards LD, Lomas DA, et al. 2014. Characterization of COPD heterogeneity in the ECLIPSE cohort. Respir Res. 11(1):122. Disponible en: https:// link.springer.com/article/10.1186/1465-9921-11-122

Agustí, A., \& Faner, R. COPD beyond smoking: new paradigm, novel opportunities. 2018. The Lancet Respiratory Medicine. 6(5):324-6. Disponible en: https://www.thelancet.com/journals/ lanres/article/PIIS22 13-2600(18)30060-2/fulltext?\%7B \$trackingTag\%7D=

Casas Maldonado, F., Arnedillo Muñoz, A., \& López-Campos, J.L. 2017. Documento de recomendaciones para el diagnóstico y tratamiento de la enfermedad pulmonar obstructiva crónica en Andalucía. Rev Esp Patol Torac. 29(2)Suplemento 2:5-24. Disponible en: http://www. neumosur.net

Cosio B.G., Soriano J.B, López-Campos J.L., Calle M. Soler J.J, de-Torres J.P, et al. 2016. CHAIN study. Distribution and outcomes of a phenotype-based approach to guide COPD management: Results from the CHAIN cohort. PLoS One. 11(9), e0160770. Disponible en: https://journals. plos.org/plosone/article?id=10.1371/journal.pone.0160770

De Abajo AB., Méndez E., González-Gallego J., Capón J., Díaz D., Peleteiro B., Mahmoud O., et al. Estimación de la prevalencia de los fenotipos en pacientes con enfermedad pulmonar obstructiva crónica. Estudio ADEPOCLE. Nutr Hosp. [Internet]. 2017 [citado 2017 Nov 17]; 34 (1):180-5. Disponible en: http://dx.doi.org/10.20960/nh.995

de Oca, M. M., Halbert, R. J., Lopez, M. V., Perez-Padilla, R., Tálamo, C., Moreno, D., et al. 2012. The chronic bronchitis phenotype in subjects with and without COPD: the PLATINO study. Eur Respir J; 40(1):28-36. Disponible en: https://erj.ersjournals.com/content/40/1/28.short

García, S. G., Fernández, L. C., García, J. J., \& Rilo, J. C. N. 2017. Enfermedad pulmonar obstructiva crónica: los pacientes en la vida real. Estudio LEONPOC. Atención Primaria. 49(10):603-10. Disponible en: https://www.sciencedirect.com/science/article/pii/S02 12656716305364

Golpe RP., López SJ., Cano Jiménez E., Castro O., Pérez de Llano LA. 2014. Distribución de fenotipos clínicos en pacientes con enfermedad pulmonar obstructiva crónica por humo de biomasa y por tabaco. Arch Bronconeumol. 50(8):318-24. Disponible en: https://doi.org/10.1016/j. arbres.2013.12.013

Izquierdo, J. L., Miravitlles, M., Esquinas, G., Pérez, M., Calle, M., Campos, J. L. L., et al. 2017. Características de los pacientes con EPOC tratados en neumología en España según grupos GOLD y fenotipos clínicos GesEPOC. Arch Bronconeumol. Disponible en: https://www.sciencedirect. com/science/article/pii/S0300289618301765

Kania, A., Krenke, R., Kuziemski, K., Czajkowska-Malinowska, M., Celejewska-Wojcik, N., KuźnarKamińska, B., et al. 2018. Distribution and characteristics of COPD phenotypes - results from the Polish sub-cohort of the POPE study. Int J Chron Obstruct Pulmon Dis. 13:1613-21. Disponible en: https://www.ncbi.nlm.nih.gov/pmc/articles/PMC5963485/

Legrá N, Grave de Peralta YT, Riverón I, del Campo E, Silvera S. 2014. Caracterización clínico epidemiológica de pacientes con enfermedad pulmonar obstructiva crónica. MEDISAN.18:1274. Disponible en: https://www.medigraphic.com/cgi-bin/new/resumen.cgi?IDARTICULO=53648 
Martínez Pérez JA., Vásquez Martín CE., Rodríguez Zapata M. 2016.Prevalencia de la enfermedad pulmonar obstructiva crónica en una zona rural de Guadalajara. Rev Esp Salud Pública; 90(5): 1-10. Disponible en: http://scielo.isciii.es/pdf/resp/v90/1135-5727-resp-90-e40002.pdf

Miravitlles M., Calle M., Soler-Cataluña JJ. 2012.Fenotipos clínicos de la EPOC. Identificación, definición e implicaciones para las guías de tratamiento. Arch. Bronconeumol;48(3): 86-98. Disponible en: https://doi.org/10.1016/j.arbres.2011.10.007

Miravitlles M., Soler-Cataluña JJ., Calle M., Molina J., Almagro P., Quintano JA., Riesco JA., Trigueros JA. et al. 2014. Guía española de la EPOG (GesEPOG). Actualización 2014. Arch. Bronconeumol; 50(1):1-16. Disponible en: http://www.archbronconeumol.org/es/guia-espanolaEPOG-gesEPOG actualizacion/articulo/90268739/

Morales Menéndez M.; Morales Menéndez M. 2015. Perfil epidemiológico-clínico de pacientes con enfermedad pulmonar obstructiva crónica en el Centro de Salud Jardinillos. AMC; 19 (2). Disponible en: http://scielo.sld.cu/scielo.php?script=sci_arttext\&pid=S1025-02552015000200004

Ntritsos, G., Franek, J., Belbasis, L., Christou, M. A., Markozannes, G., Altman, P., et al. 2018. Genderspecific estimates of COPD prevalence: a systematic review and meta-analysis. International journal of chronic obstructive pulmonary disease; 13:1507-14. Disponible en: https://www.ncbi. nlm.nih.gov/pmc/articles/PMC5953270/

Reiger G, Zwick R, Lamprecht B, Kahler C, Burghuber OC, Valipour A. 2018. Phenotypes of COPD in an Austrian population. Wien Klin Wochenschr; 130:382-9. Disponible en: https://link.springer. com/article/10.1007/s00508-018-1347-7

Robainas del Pino I, García Silvera E. 2020. Comparación clínica y funcional entre pacientes con diagnóstico de enfermedad pulmonar obstructiva crónica severa y muy severa. Rev Cubana Med; 58(4). Disponible en: http://revmedicina.sld.cu/index.php/med/article/view/1313

Rubio, M. C., Casamor, R., \& Miravitlles, M. 2017. Identification and distribution of COPD phenotypes in clinical practice according to Spanish COPD guidelines: the FENEPOC study. Int J Chron Obstruct Pulmon Dis; 12:2373-83. Disponible en: https://www.ncbi.nlm.nih.gov/ pubmed/28848338

Ruiz, C. A. J., Buljubasich, D., Miranda, J. A. R., Izcaray, A. A., de Granda Orive, J. I., Chatkin, J. M., et al. 2017. Preguntas y respuestas relacionadas con tabaquismo en pacientes con EPOC. Aplicación de metodología con formato PICO. Archivos de Bronconeumología; 53(11):622-8. Disponible en: https://www.sciencedirect.com/science/article/pii/S0300289617301333

Sáenz, C. B. 2017. Enfermedad Pulmonar Obstructiva Crónica (EPOC) y tabaquismo. Revista de la Asociación Médica Argentina; 130(3):35-6. Disponible en: https://www.ama-med.org.ar/ uploads_archivos/1357/Rev-3-2017-Pag-35-Saenz.pdf

Vinaccia Alpi S., Riveros Munévar F., Quiceno Sierra J. 2017. Análisis exploratorio del Airways Questionnaire 20 (AQ20) en pacientes colombianos con enfermedad pulmonar obstructiva crónica. Rev. am. med. respir; 17 (1). Disponible en: https://www.redalyc.org/pdf/3821/382150406009. pdf

Vidalón-Soldevilla E, Ventura-Fernández G, Torres-Godomar R. 2011. Características de la enfermedad pulmonar obstructiva crónica en fumadores y exfumadores atendidos en un hospital de la provincia de Ica, Perú. Rev Med Panacea; 1:67-71. Disponible en: https://revistas.unica.edu. pe/index.php/panacea/article/view/108 


\begin{abstract}
Introduction: The identification of clinical phenotypes are key in the modulation of clinical expression, for an integrated treatment of COPD. Objectives: To characterize the clinical phenotypes of COPD in patients treated at the Hospital Neumológico Benéfico Jurídico. Methods: A retrospective descriptive observational study was carried out in 172 patients with a diagnosis of COPD at the Hospital Neumológico Benéfico Jurídico in 2017. Results: 38.4\% of the patients were between 70-79 years of age. Of the total number of patients, $54.6 \%$ were male. $52.9 \%$ were active smokers and $41.3 \%$ ex-smokers. Although the differences were not significant, advanced age and male sex were more frequent in the exacerbator emphysematous and chronic bronchial exacerbator phenotype. Active smoking was more frequent in the exacerbating emphysematous phenotype. All patients with the chronic bronchial exacerbator phenotype had two or more exacerbations, while exacerbation emphysematous was associated with severe severity of COPD (46.7\%). Conclusions: Male sex and advanced age show a tendency to be related to the exacerbating emphysematous phenotype and chronic bronchitis exacerbator, while active smoking is more frequent in the exacerbating emphysematous phenotype. The chronic bronchitis exacerbator phenotype is related to greater exacerbations and exacerbation emphysematous with a greater severity of COPD.
\end{abstract}

Keywords: COPD; clinical phenotypes of chronic obstructive pulmonary disease; Clinical epidemiology 